\title{
Meige Syndrome: An Eternal Diagnostic Confusion
}

\author{
Amit Chauhan', Shravani Chauhan ${ }^{2 *}$ \\ ${ }^{1}$ Department of Orthopedics, Park Hospital, Gurgaon, Haryana, India \\ ${ }^{2}$ Department of Psychiatry, VIMHANS, New Delhi, India \\ Email: shrav1980@gmail.com
}

Received 13 May 2015; accepted 7 July 2015; published 10 July 2015

Copyright (C) 2015 by authors and Scientific Research Publishing Inc.

This work is licensed under the Creative Commons Attribution International License (CC BY).

http://creativecommons.org/licenses/by/4.0/

(c) (i) Open Access

\begin{abstract}
Meige syndrome is an idiopathic dystonia characterized by combination of blepharospasm and involuntary movements of the lower facial and/or masticatory (jaw) muscles. The condition is rare and has a variety of clinical presentations which often lead to its misdiagnosis. We report a case of Meige syndrome repeatedly misdiagnosed and treated unsuccessfully as conversion disorder.
\end{abstract}

\section{Keywords}

\section{Meige Syndrome, Orofacial Dystonia, Diagnostic Confusion}

\section{Introduction}

Meige syndrome is an idiopathic dystonia characterized by symmetrical blepharospasm and oromandibular dystonia [1]. It is used synonymously with Brueghel's syndrome or segmental craniocervical dystonia. Although the causes of this syndrome are unknown, it has been reported to be induced by certain kinds of drugs such as antipsychotic drugs and dopamine agonists, cerebellar degeneration, basal ganglia dysfunction, and brain tumors [2]. The condition is rare and has clinical presentations like abnormal blinking, squinting/eyes closing during speech, trismus, clenching or grinding of teeth, lip tightening and pursing, deviation or protrusion of the tongue and drawing back of corners of the mouth. In some patients, dystonic spasms may be provoked by certain activities, such as talking, chewing, or biting. The variety of symptoms and low prevalence of the syndrome often leads to its misdiagnosis in psychiatry, ophthalmology and neurology. We report a case of Meige syndrome repeatedly misdiagnosed and treated as conversion disorder.

*Corresponding author. 


\section{Case Report}

Mrs S., a 60-year-old lady, presented in orthopedic outpatient department with 3-year complaint of tightening of oral, facial and neck muscles along with neck pain for last 3 months. After initial assessment for cervical spondylosis, the orthopedician referred her to psychiatry for evaluation of the abnormal facial grimacing movements. On detailed history taking, patient revealed that symptoms started acutely 3 years back with repeated blinking of eyes. There were no apparent precipitating factors, no history of medication intake with negative family history of neurological and psychiatric illness and no comorbid medical illness. She was initially shown to an ophthalmologist who did not prescribe any specific medication. Two to three months after onset of increased eye blinking, patient started having painless tightening of neck muscles and subsequent upward deviation of face and neck, not relieved by massage or rest. This was followed by difficulty in opening her mouth (trismus) along with clenching and grinding of teeth (bruxism) whenever she tried to speak. Within six months, patient started having difficulty in opening her mouth to eat food along with protrusion of tongue and increased frequency of neck muscle tightening leading to bizarre grimacing of the face. She was referred to a psychiatrist, who diagnosed her as conversion disorder and prescribed amitriptylline (50 mg/day), haloperidol (10 mg/day) and trihexyphenidyl (4 mg/day). Two months after medication, there was no improvement so she was referred for neurological opinion. Routine blood tests and imaging (CT scan) were normal. Patient was confirmed as a case of conversion disorder and prescribed fluoxetine $(20 \mathrm{mg} /$ day $)$, olanzapine $(10 \mathrm{mg} /$ day $)$ and trihexyphenidyl $(4 \mathrm{mg} /$ day). She took above medication for few months and subsequently discontinued as symptoms worsened. Disappointed with treatment outcome, patient stopped taking medication for next one year during which her symptoms continued.

For last three months, patient started feeling pain in her neck during movement, especially during neck flexion. For this reason, she consulted an orthopedic surgeon, who referred her to psychiatric outpatient department. On clinical examination, patient presented with uncontrollable closing of eyes (blepharospasm), upward deviation of neck, grimacing of face, trismus and bruxism along with inability to speak properly due to neck muscle spasm. After thorough neurological and psychiatric check up, a diagnosis of idiopathic craniocervical dystonia/ Meige syndrome was made and patient started on baclofen, a GABA-agonist drug. The drug was started at 20 $\mathrm{mg} /$ day, increased by $10 \mathrm{mg}$ every three days reaching a maximum dose of $60 \mathrm{mg} /$ day. There was gradual improvement in trismus, bruxism and dysarthria, though blepharospasm showed minimal improvement. After three months of follow up, patient reported improved quality of life but continued to have eye symptoms and occasional neck and jaw spasms.

\section{Discussion}

Historically, Dr. Horatio Wood, a Philadelphia neurologist, first drew attention to blepharospasm and other cranial dystonias in 1887 [3]. In 1910, Dr. Henri Meige, a French neurologist, described ten patients with involuntary closure of the eyelids [1]. More than half a century later, an American neurologist, George Paulson, reported three patients with blepharospasm and oromandibular dystonia and emphasized the probability of a common pathophysiological basis [4]. Though studied for a long time, the cause of Meige syndrome still remains elusive and treatment remains debatable. Basal ganglion dysfunction has been postulated with involvement of dopamine receptors [5]. In the nigro-striatal pathway, one of the retrograde loops in the feed-back control of dopamine synthesis by nigral neurons is dependent on GABA. Increasing GABA activity through GABA agonists that cross the blood-brain barrier could result in a decreased dopaminergic action in the nigro-striatal pathway and, thus, ameliorate the dystonic symptoms which might have been produced by its increased function [5]. Baclofen, a GABA agonist, has been tried in dystonias for the above reason and has shown variable results. Other medical treatments like anti cholinergics, botulinum toxin injections and benzodiazepines usually present disappointing results. Surgical treatments include bilateral deep brain stimulation of the globus pallidus internus which has become a first choice treatment for drug refractory, primary, segmental, or generalized dystonias [6].

The authors report no proprietary or commercial interest in any product mentioned or concept discussed in this article.

\section{References}

[1] Meige, H. (1910) Les convulsions de la face, une forme clinique deconvulsion faciale, bilatérale et médiane. Revista de 
Neurología, 20, 437-443.

[2] Hayashi, T., Furutani, M., Taniyama, J., et al. (1998) Neuroleptic Induced Meige’s Syndrome Following Akathisia: Pharmacologic Characteristics. Psychiatry and Clinical Neurosciences, 52, 445-448. http://dx.doi.org/10.1046/j.1440-1819.1998.00408.x

[3] Wood, H.C. (1887) Nervous Diseases and Their Diagnosis: A Treatise upon the Phenomena Produced by Diseases of the Nervous System. JB Lippincott, Philadelphia, 1937.

[4] Paulson, G.W. (1972) Meige’s Syndrome. Dyskinesia of the Eyelids and Facial Muscles. Geriatrics, 8, 69-73.

[5] De Andrade, L.A. and Bertolucci, P.H. (1985) Treatment of Meige Disease with a GABA Receptor Agonist. Arquivos de Neuro-Psiquiatria, 43, 260-266. http://dx.doi.org/10.1590/S0004-282X1985000300004

[6] Reese, R., Gruber, D., Schoenecker, T., et al. (2011) Long-Term Clinical Outcome in Meige Syndrome Treated with Internal Pallidum Deep Brain Stimulation. Movement Disorders, 26, 691-698. http://dx.doi.org/10.1002/mds.23549 